\title{
Improved next-generation sequencing pre-capture library yields and sequencing parameters using on-bead PCR
}

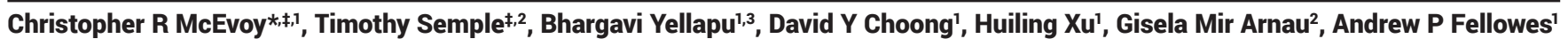
\& Stephen B Fox'

\begin{abstract}
Tumor DNA sequencing results can have important clinical implications. However, its use is often limited by low DNA input, owing to small tumor biopsy size. To help overcome this limitation we have developed a simple improvement to a commonly used next-generation sequencing (NGS) capture-based library preparation method using formalinfixed paraffin-embedded-derived tumor DNA. By using on-bead PCR for pre-capture library generation we show that library yields are dramatically increased, resulting in decreased sample failure rates. Improved yields allowed for a reduction in PCR cycles, which translated into improved sequencing parameters without affecting variant calling. This methodology should be applicable to any NGS system in which input DNA is a limiting factor.
\end{abstract}

\section{LAY ABSTRACT}

The results of tumor DNA sequencing can have important clinical implications. However, limited DNA input owing to small tumor size frequently results in a failed analysis. By incorporating a simple modification to the standard sample preparation method, we show that DNA yields can be greatly increased, allowing for more samples to be successfully sequenced. Further, the methodology used also results in a subsequent improvement in sequencing parameters, with no loss in the ability to detect mutations.

\section{METHOD SUMMARY}

To improve the library yield for low DNA input tumor samples subjected to NGS, we adopted a simple modification of the standard KAPA Hyper library preparation procedure. Genomic DNA libraries were subjected to a solid-phase reversible mobilization bead-based cleanup; however, they were not eluted from the beads prior to pre-capture PCR. Post-PCR cleanup was performed using a $20 \%$ PEG, $2.5 \mathrm{M} \mathrm{NaCl}$ buffer, resulting in a dramatic yield increase with no loss of sequencing fidelity.

\section{KEYWORDS}

DNA library $\cdot$ next-generation sequencing $\cdot$ solid-phase reversible immobilization beads

'Department of Pathology, Peter MacCallum Cancer Centre, Melbourne, VIC 3000, Australia; ${ }^{2}$ Cancer Research Division, Peter MacCallum Cancer Centre, Melbourne, VIC 3000, Australia; ${ }^{3}$ Department of Molecular Oncology \& Cancer Immunology, Epworth Healthcare, Melbourne, VIC 3121, Australia; *Author for correspondence: christopher.mcevoy@petermac.org; $¥$ Joint first authors

BioTechniques 68: 48-51 (January 2020) 10.2144/btn-2019-0059
Tumor DNA analysis using next-generation sequencing (NGS) has revolutionized many aspects of cancer diagnosis, prognosis, and therapeutics [1]. Two major factors, DNA quality and quantity, influence the proportion of successfully sequenced tumor samples. The quality issue is attributed to the use of formalin-fixed paraffin-embedded (FFPE) tumor tissue, which results in highly fragmented DNA that is chemically modified, whereas the low quantity results from small tumor biopsy sizes. In our experience, using the KAPA Hyper Prep Kit for library preparation (Roche, Basel, Switzerland), in conjunction with a SureSelect ${ }^{\mathrm{XT}}$ targeted capture (Agilent, CA, USA), we found that approximately 15\% (61/432) of the tumor samples failed to produce a sufficient yield of pre-capture library. Therefore, we modified the standard protocol by performing the pre-capture PCR 'on-bead'. Solid-phase reversible immobilization (SPRI) bead-based DNA purification [2] has become the favored method of DNA purification in many protocols owing to its ease of use, cost-effectiveness, ability to size-select, and suitability for automation. Following genomic DNA (gDNA) fragmentation and adapter ligation, NGS capture-based libraries typically undergo SPRI bead-based purification in which the DNA eluted from the beads is used for PCR enrichment. This elution step inevitably results in the loss of DNA. This study was designed to determine whether performing the PCR directly on the bead/eluate mixture resulted in improved pre-capture library yields when using FFPE-derived tumor samples.

A detailed protocol for library preparation can be found in the Supplementary data file. Tumor DNA was extracted from FFPE sections using a QIAamp DNA FFPE Tissue Kit (Qiagen, Hilden, Germany) and was fragmented using a LE220 focused-ultrasonicator (Covaris, MA, USA). DNA libraries were then prepared using the KAPA Hyper Prep Kit, per the manufacturer's instructions (Roche) with some modifications. Following DNA end-repair and A-tailing, SureSelect adapters (Agilent) were ligated and SPRI (AMPure, Beckman Coulter, IN, USA) bead purification was performed. However, no final elution of purified DNA was performed. Instead, the entire bead/elution buffer mixture was added to the KAPA PCR mixture where it underwent normal PCR cycling. Therefore, amplification of the pre-capture gDNA library occurred in the presence of the beads. This process is illustrated in Figure 1. Following PCR, the amplified library was purified by adding $90 \mu \mathrm{l}$ of $20 \% \mathrm{PEG} / 2.5 \mathrm{M} \mathrm{NaCl}$ [2], instead of the recommended $90-\mu \mathrm{I}$ SPRI bead mixture. Its addition "reactivates" the beads that are already present, and bead-based purification can continue as normal. Following purification, pre-capture libraries were analyzed for yield and average fragment size (range 100-1000 bp) using a 4200 TapeStation System (Agilent). SureSelect ${ }^{\mathrm{XT}}$ hybridization using a custom 386 gene, 2.34 Mb probe set was used for target enrichment. The capture of hybridized fragments and their washes and final purification was performed as per the standard SureSelect ${ }^{\mathrm{xT}}$ protocol. Pooled libraries were sequenced on an Illumina NextSeq500 (Illumina, CA, USA) using paired 75 bp reads. 


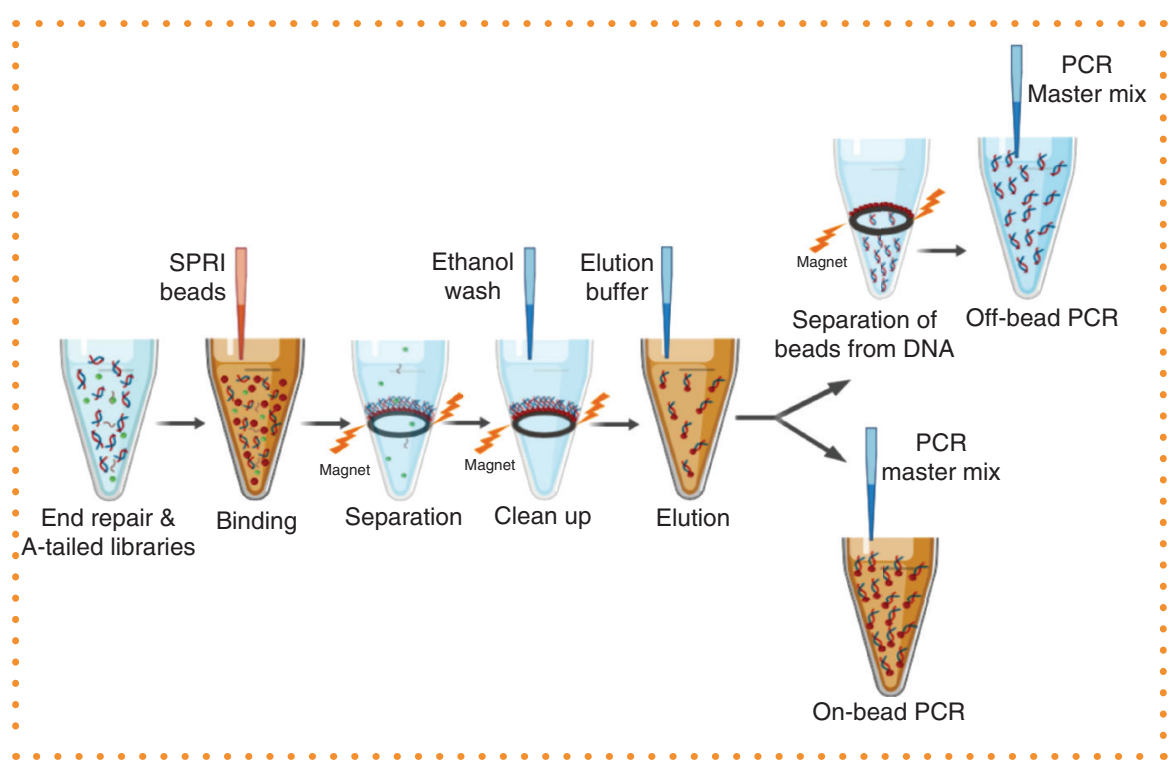

Figure 1. Overview of the methods used in the study. SPRI: Solid-phase reversible immobilization.
We first sought to determine whether pre-capture library yields were affected by using the on-bead PCR method. One highquality cell line-derived DNA (NA12878, Coriell Institute) and one FFPE-derived tumor DNA were used, each at two different input amounts. The standard 'off-bead' and modified 'on-bead' methods were performed in parallel for the four samples. The results revealed a dramatic increase in pre-capture library yield in each case, with an average increase of 4.15-fold (Figure 2A).

We next compared pre-capture library yields between the off-bead method using the standard 10 PCR cycles and the on-bead method using nine PCR cycles. Our rationale was that if sufficient on-bead yields could still be generated with one less PCR cycle, the sequencing read duplicates would be reduced, leading to an improved total read depth for these samples. To test this
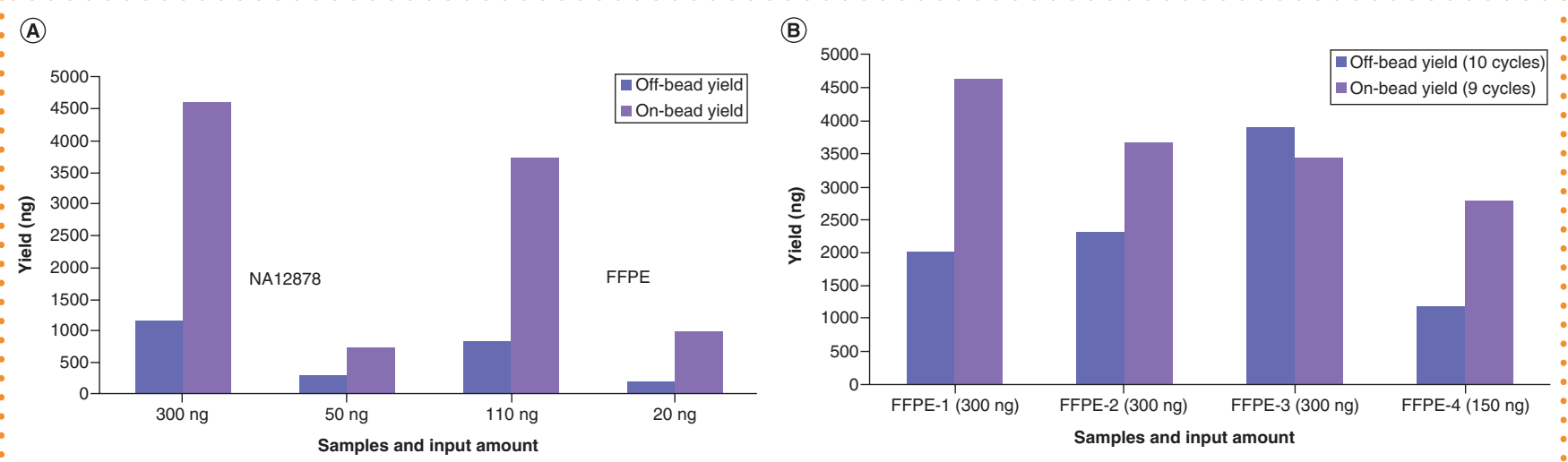

(C)

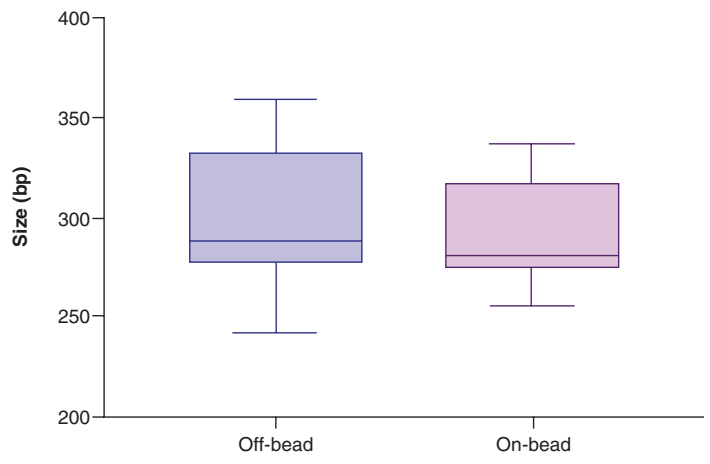

(D)

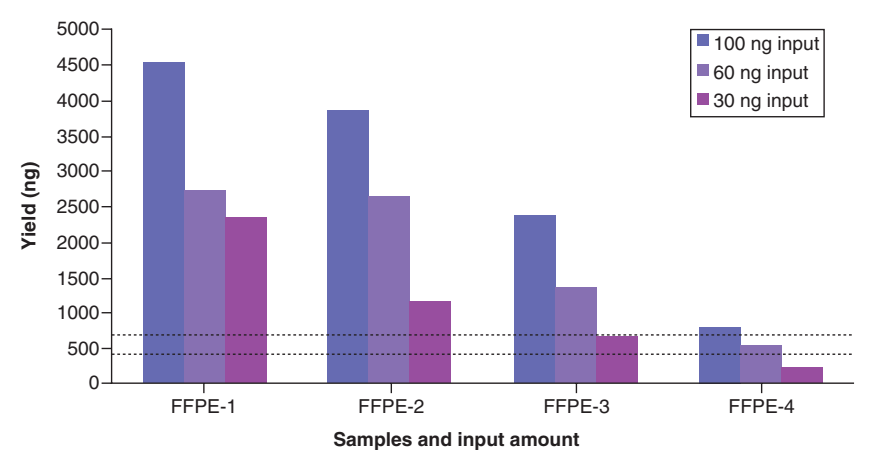

Figure 2. Analysis of off-bead versus on-bead pre-capture library preparations. (A) Library yields for one control and one FFPE tumor sample performed at two different DNA input amounts. All four sample preparations were performed in parallel. Average yield increase using on-bead PCR was 4.15-fold. (B) Off-bead versus on-bead yield comparisons for four FFPE tumor samples, with off-bead samples undergoing 10 PCR cycles and on-bead samples undergoing nine cycles. On-bead yields increased an average of 1.8-fold despite the reduction in cycles. (C) Box plots of average fragment sizes for the eight comparisons used in (A \& B). On-bead PCR did not appear to significantly impact on library fragment size (Paired $t$-test, $p=0.122, t=1.76$, $\mathrm{df}=7$ ). (D) Analysis of DNA input limits required to generate a pre-capture library yield sufficient for hybridization when using nine cycles on-bead PCR. Recommended $(750 \mathrm{ng})$ and lower limit $(500 \mathrm{ng}$ ) yields are shown by dashed horizontal lines. Results show that $30 \mathrm{ng}$ of input generates sufficient library in two cases, a borderline yield in one case and a failed library in one case.

FFPE: Fomalin-fixed paraffin-embedded. 
Table 1. Selected NGS parameters from four sample pairs.

\begin{tabular}{|l|l|l|l|l|l|l|l|l|l|l|}
\hline Sample_ID & FFPE-1 Off & FFPE-1 On & FFPE-2 Off & FFPE-2 On & FFPE-3 Off & FFPE-3 On & FFPE-4 Off & FFPE-4 On \\
\hline Mean coverage & 209.7 & 383.0 & 355.9 & 426.4 & 302.9 & 439.6 & 344.5 \\
\hline \% bases above 100 & 93.5 & 98.1 & 93.2 & 94.9 & 95.7 & 97.6 & 95.7 \\
\hline \% bases above 500 & 0.6 & 20.8 & 22.2 & 33.9 & 11.3 & 30.9 & 16 & 97.4 \\
\hline Total reads & $34,741,134$ & $36,859,984$ & $41,782,784$ & $36,957,886$ & $31,148,930$ & $35,678,966$ & $39,477,926$ & $37,762,250$ \\
\hline $\begin{array}{l}\text { \% Mapped reads } \\
\text { duplicates }\end{array}$ & 38.53 & 23.76 & 35.76 & 23.22 & 26.39 & 9 & 37.38 & 21.01 \\
\hline $\begin{array}{l}\text { Total reads minus } \\
\text { duplicates }\end{array}$ & $21,414,277$ & $28,141,701$ & $26,891,969$ & $28,405,461$ & $22,970,169$ & $32,485,748$ & $24,774,921$ & $29,853,605$ \\
\hline \% Reads on target & 35.0 & 48.0 & 51.4 & 56.0 & 47.2 & 47.3 & 48.3 \\
\hline $\begin{array}{l}\text { Mean coverage for } \\
\text { target bases }\end{array}$ & 246.6 & 450.4 & 462.3 & 536.8 & 359.5 & 511.9 & 399.8 \\
\hline $\begin{array}{l}\text { Median fragment } \\
\text { length }\end{array}$ & 144 & 142 & 113 & 121 & 137 & 138 & 56.5 \\
\hline
\end{tabular}

Note the reduction in '\% Mapped read duplicates' attributed to the reduction in cycles for the on-bead samples and the corresponding increase in read depth and coverage statistics. Median fragment length remains unchanged.

FFPE: Formalin-fixed paraffin-embedded; NGS: Next-generation sequencing.

hypothesis, four FFPE tumor DNAs were prepared in parallel. Three sample pairs used 300 ng of input DNA, whereas one used $150 \mathrm{ng}$. Figure $2 \mathrm{~B}$ shows that in three of the four pairs the on-bead method, with nine PCR cycles, produced a greater library yield than the standard off-bead method with 10 cycles. The average on-bead increase in yield across the four sample pairs was 1.8-fold. In addition, we compared the average fragment size generated for these four sample pairs, along with the four sample pairs tested in the first analysis (Figure 2A). On-bead PCR did not appear to impact on the library fragment size ( $p=0.12$, paired $t$-test) (Figure $2 C$ ).

Sequencing libraries for the four sample pairs from Figure 2B were prepared and sequenced in a single run. Table 1 shows nine important sequencing metric comparisons from the four pairs. In each case, the on-bead samples had a lower '\% mapped reads duplicates' value, whereas all read depth and coverage statistics were improved despite two of the off-bead samples having a higher 'total reads' value. Median fragment length was unchanged. All four of these tumor samples, plus the tumor sample analyzed in test 1 (Figure 2A), had previously been analyzed with our 386 gene targeted panel under standard conditions. Therefore, we compared the variants detected in our on-bead results with those previously detected. These tumor samples had been selected because of the high number and diversity of their variants. A total of 66 variants had been detected in these five samples. These comprised $60 \times$ SNVs, $1 \times 1$ bp deletion, $1 \times 1$ bp insertion, $1 \times 8$ bp deletion, $1 \times 24$ bp deletion, $1 \times 25$ bp deletion, and $1 \times$ gene fusion (GOLGA4-RAF1). All 66 variants were detected in our on-bead samples, and variant allele frequencies were similar ( $t$-test for two independent means, $p$ $=0.21$ ) (Supplementary Table 1).

Finally, we sought to determine the lower limits of DNA input required to produce sufficient pre-capture library for hybridization. The SureSelect ${ }^{\mathrm{XT}}$ protocol recommends using $750 \mathrm{ng}$ of pre-capture library, although we found a lower limit of $500 \mathrm{ng}$ is sufficient. Accordingly, we designated pre-capture library yields $>750 \mathrm{ng}$ as 'pass', 750-500 ng as 'borderline' and $<500$ ng as 'fail'. Four FFPE tumor DNAs were used, each at gDNA inputs of 100, 60 and $30 \mathrm{ng}$. Pre-capture libraries were produced using the on-bead method using nine PCR cycles. Results show that at $100 \mathrm{ng}$ input all samples passed, at $60 \mathrm{ng}$ input three samples passed and one was borderline, and at $30 \mathrm{ng}$ input two samples passed, one sample was borderline, and one sample failed (Figure 2D). We are able to obtain $>30$ ng of DNA from $>95 \%$ of our FFPE tumor samples. This represents a significant increase in likely 'passes' compared with our previous results, in which approximately $15 \%$
$(61 / 432)$ of the samples failed to generate sufficient library for hybridization. Note that this is despite a reduction in PCR cycles.

In summary, we demonstrate that the use of on-bead pre-capture library enrichment dramatically increased our library yields. The concept of skipping SPRI eluting steps to gain efficiency has been previously described in detail by Fisher et al. [3] and termed 'with-bead SPRI'. Fisher describes the use of with-bead SPRI after end-repair, A-tailing and adaptor ligation. However, using the KAPA Hyper Prep Kit these steps are already optimized in a single tube; our work shows further efficiency gains by directly doing an on-bead PCR. In our laboratory this has resulted in approximately $50 \%$ fewer FFPE tumor samples failing sequencing. Furthermore, the protocol enables a decrease in the number of PCR cycles used and this has had a positive effect on the final sequencing results, with less read duplicates, and higher read depths and coverage noted. Crucially, as a diagnostic laboratory analyzing patient tumor samples, it has had no negative effect on variant calling accuracy or variant allele frequency determination. This simple modification of a well-established protocol should be applicable to many other NGS systems and, indeed, many other methodologies where a pre-PCR bead-based clean-up is required on limited amounts of input DNA. 
Here, we present a simple yet effective method for improving PCR yields and sequencing parameters, following SPRI bead-based cleanups of NGS pre-capture libraries. This should aid with increasing the numbers of clinical samples that are able to be sequenced and provide subsequent clinical benefit for patients. Although we have used this method in an NGS setting, it should be applicable to any case in which limited DNA is available or higher PCR yields are required.

\section{SUPPLEMENTARY DATA}

To view the supplementary data that accompany this paper please visit the journal website at: www.future-science. com/doi/suppl/10.2144/btn-2019-0059

\section{AUTHOR CONTRIBUTIONS}

TS, CRM, GM, HX, SBF and APF conceived the experiments. CRM, BY and DYC performed all of the experiments. CRM and $\mathrm{GM}$ analyzed the results. CRM wrote the paper with assistance from all co-authors.

\section{ACKNOWLEDGMENTS}

Tumor profiling at Peter Mac is made possible by the generous support of the Peter MacCallum Foundation.

\section{ETHICAL CONDUCT OF RESEARCH}

The authors state that they have obtained appropriate institutional review board approval.

\section{FINANCIAL \& COMPETING}

\section{INTERESTS DISCLOSURE}

The authors have no relevant affiliations or financial involvement with any organization or entity with a financial interest in or financial conflict with the subject matter or materials discussed in the manuscript. This includes employment, consultancies, honoraria, stock ownership or options, expert testimony, grants or patents received or pending, or royalties.

No writing assistance was utilized in the production of this manuscript.

\section{OPEN ACCESS}

This work is licensed under the AttributionNonCommercial-NoDerivatives 4.0 Unported License. To view a copy of this license, visit http://creativecommons.org/licenses/ by-nc-nd/4.0/

\section{REFERENCES}

1. Sabour $L$, Sabour M, Ghorbian S. Clinical applications of next-generation sequencing in cancer diagnosis. Pathol. Oncol. Res. 23(2), 225-234 (2017).

2. DeAngelis MM, Wang DG, Hawkins TL. Solid-phase reversible immobilization for the isolation of PCR products. Nucleic Acids Res. 23(22), 4742-4743 (1995).

3. Fisher S, Barry A, Abreu J et al. A scalable, fully automated process for construction of sequence-ready human exome targeted capture libraries. Genome Biol. 12(1), r1 (2011). 